\title{
ARTICLE
}

Epidemiology

\section{Risk of cardiovascular events in men treated for prostate cancer compared with prostate cancer-free men}

\author{
Ida Rask Moustsen (iD), Signe Benzon Larsen ${ }^{1,2}$, Anne Katrine Duun-Henriksen ${ }^{3}$, Anne Tjønneland ${ }^{4,5}$, Susanne K. Kjær ${ }^{6}$, Klaus Brasso $^{2}$, \\ Christoffer Johansen ${ }^{7,1}$ and Susanne Oksbjerg Dalton ${ }^{1,8}$
}

BACKGROUND: The effect of lifestyle, anthropometry and cardiovascular risk factors on cardiovascular disease in men with prostate cancer $(\mathrm{PCa})$ remains unclear.

METHODS: Using a population-based cohort of 25,436 Danish, cancer-free men aged 50-64 years, we obtained information on selfreported pre-cancer lifestyle, objectively measured anthropometry and cardiovascular risk factors, and linked them to national health registers for information on major cardiovascular outcomes. We assessed hazard ratios (HRs) of incident acute myocardial infarction (MI), ischaemic stroke (IS) and heart failure (HF) among 1546 men diagnosed with PCa treated with first-line active surveillance, watchful waiting, intended curative or palliative treatment compared with PCa-free men during 18 years of follow-up. RESULTS: Men who received first-line palliative treatment had higher rates of IS and HF with adjusted $\mathrm{HRs}$ of $2.09(95 \% \mathrm{CI}$ 1.49-2.93) and 2.05 (95\% Cl 1.43-2.94), respectively, compared with PCa-free men. The risks were increased from start of treatment. We did not find the same relation for men in any other treatment group. No differences between men treated for PCa and cancerfree controls were observed for $\mathrm{Ml}$ after adjustment for lifestyle, anthropometry, and cardiovascular risk factors.

CONCLUSION: Pre-diagnosis lifestyle, anthropometry or cardiovascular risk factors did not explain the risk of IS and HF in PCa patients receiving palliative treatment. The results emphasise the need for balancing disease management and monitoring of cardiovascular health in this patient group.

British Journal of Cancer (2019) 120:1067-1074; https://doi.org/10.1038/s41416-019-0468-8

\section{BACKGROUND}

Since the 90s, the incidence of prostate cancer (PCa) has increased threefold in Denmark as it has elsewhere. ${ }^{1-3}$ This is mainly caused by better screening and the majority of men are diagnosed with localised and potentially curable disease resulting in good survival prospects for most patients. ${ }^{4,5}$ Currently, more than 33,000 Danish men are living with PCa comprising the largest group of male cancer survivors. ${ }^{1}$ Among these, a number will experience disease progression and face the consequences of cancer-targeting treatments.

A well-investigated late effect of PCa treatment is cardiovascular (CV) disease. The suggested biological mechanisms include a direct cardio-toxic effect of hormone manipulation or an indirect CV effect through metabolic mechanisms. ${ }^{6-13}$ Review of the literature show inconsistent results on the association between PCa and risk of CVD, which may relate to differences in age, stage, length of follow-up, type of therapy and choice of CV outcomes, which vary widely across studies. Further, few studies have compared the risk of CV outcomes for PCa patients with that of PCa-free men and with different results. ${ }^{14,15}$ Armenian et al. ${ }^{14}$ found a lower CV risk (incidence rate ratio (IRR), $0.89 ; 95 \%$ confidence interval $(\mathrm{Cl}) 0.84-0.95$ ) compared with cancer-free men, whereas O'Farrell et al. ${ }^{15}$ found an increased risk, hazard ratio (HR) $1.21(95 \% \mathrm{Cl}$ $1.18-1.25)$.

When investigating the risk of CV events after diagnosis, it is paramount to address pre-diagnostic lifestyle and pre-existing CV risk to elucidate the complex relationship between existing risk factors at time of diagnosis and initiation of anti-cancer treatment and treatment-induced morbidity. ${ }^{16}$ Smoking, high-risk drinking and a sedentary lifestyle are factors associated with the risk of CV disease, as are a high body mass index (BMI) and waist circumference, as well as hypertension, hypercholesterolaemia and diabetes. ${ }^{17-20}$

We aimed to investigate risk differences of major ischaemic disease outcomes-acute myocardial infarction (MI), ischaemic stroke (IS) and heart failure (HF) - for men with PCa in either active surveillance, watchful waiting, intended curative or palliative firstline treatment compared with men without a PCa diagnosis. Analyses were adjusted for pre-diagnosis lifestyle, anthropometry, blood pressure, serum cholesterol and diabetes, as well as CV precursor disease.

\footnotetext{
${ }^{1}$ Survivorship, Danish Cancer Society Research Center, Copenhagen, Denmark; ${ }^{2}$ Copenhagen Prostate Cancer Center, Department of Urology, Rigshospitalet, Copenhagen University Hospital, Copenhagen, Denmark; ${ }^{3}$ Statistics and Pharmaco-epidemiology, Danish Cancer Society Research Center, Copenhagen, Denmark; ${ }^{4}$ Diet, Genes and Environment, Danish Cancer Society Research Center, Copenhagen, Denmark; ${ }^{5}$ Department of Public Health, University of Copenhagen, Copenhagen, Denmark; ${ }^{6}$ Virus, Lifestyle and Genes, Danish Cancer Society Research Center, Copenhagen, Denmark; ${ }^{7}$ The Oncology Clinic, Finsen Center, Rigshospitalet 5073 , 2100 Copenhagen, University of Copenhagen, Copenhagen, Denmark and ${ }^{8}$ Department of Oncology, Zealand University Hospital Naestved, Naestved, Denmark Correspondence: Ida Rask Moustsen (idamou@cancer.dk)
} 


\section{METHODS}

Study population and data collection

Our study was based on data from the Danish prospective Diet, Cancer and Health cohort. Briefly, 80,996 men aged 50-64 years without previous cancer diagnoses were invited and 27,179 (34\%) participated. Baseline information was collected between December 1993 and May 1997, where all participants completed questionnaires concerning diet, lifestyle- and health-related issues, and trained staff obtained blood samples and anthropometric measurements. A detailed description of the cohort is provided elsewhere. ${ }^{21}$ The Diet, Cancer and Health study was approved by the regional ethical committees in Copenhagen and Aarhus ((KF) 11-037/01), and the present study by the Danish Data Protection Agency (2013-41-4232).

\section{Identification of PCa patients}

We used the unique personal identification number provided to all Danish residents to link the cohort to the Danish Cancer Registry with records of all cancers diagnosed in Denmark. ${ }^{22,23} \mathrm{We}$ identified 1838 men from the cohort diagnosed with incident PCa (ICD-10: C619) between study enrolment and 31 December 2013. By thoroughly reviewing hospital records, we obtained clinical information on stage, Prostate Specific Antigen (PSA) level, Gleason score and first-line treatment for all men diagnosed with PCa from 1993 to 2013. Stage was reported by the TNM classification system in the hospital records and was supplemented with information from the Danish Cancer Registry when missing in the record. To include stage of disease recorded before the introduction of TNM in 2003, we merged TNM with the previously used classification system, resulting in 'localised disease' and 'non-localised disease' (algorithm in Appendix). Gleason score and PSA level at baseline were categorised and included solely for descriptive purposes. First-line treatment was coded as follows: 'active surveillance' (surveillance with a curative intent in case of progression), 'watchful waiting' (surveillance with palliative intent in case of progression), 'intended curative' (radical prostatectomy, radiotherapy or brachytherapy) or 'palliative treatment' (hormone therapy (received by $94 \%$ in this group), chemotherapy, surgery or radiotherapy) for all PCa diagnosed from 1993 to 2013. Detailed description of the clinical information was previously reported. ${ }^{24}$

\section{Outcomes}

The outcomes were incident acute MI, IS or HF. Date of each incident CV event was obtained from the Danish National Patient Registry ${ }^{25}$ (NPR) including all in-patient hospital admissions since 1977 and outpatient contacts since 1995. We supplemented information on CV events with information from the Danish Cause of Death Registry dating back to 1970, to include fatal first-time CV events. $^{26}$

\section{Covariates obtained at baseline}

From self-reported questionnaires, we categorised education as 'short' (basic school only; < 7 years), 'medium' (high school or vocational; 7-12 years) or 'long' (higher education; $>12$ years). Smoking was included as 'never', 'former' or 'current'. Alcohol intake (g/day) was included as a continuous covariate. To measure physical activity, we calculated metabolic equivalent of task (MET) from an average of summer and winter physical activity multiplied by number of hours per week. MET-score was grouped in quartiles. From the anthropometric measures obtained by the staff at enrolment, the BMl was grouped as normal $(<25)$, overweight $^{25-30}$ or obese $(>30)$, whiereas waist circumference in centimetre was grouped in quartiles. Diastolic and systolic blood pressure $(\mathrm{mmHg})$ and serum cholesterol $(\mathrm{mMol} / \mathrm{L})$ measured at enrolment were included as continuous covariates. Blood pressure was measured at the right arm using automatic devices: TAKEDA UA 751 and UA 743 in lying position after a minimum of 5 min rest. Non-fasting total cholesterol $(\mathrm{mMol} / \mathrm{L})$ was determined in whole blood using a Lipotrend ${ }^{\circledR} \mathrm{C}$ device with Lipotrend test strips (Boehringer Mannheim).

Information on diabetes and precursor CV disease was obtained from the NPR at baseline. Diabetes was included as a yes/no covariate. The three CV precursor diseases, angina pectoris, transitional cerebral ischaemia (see definition in Supplementary Information online) and cardiomyopathy, were coded as a combined yes/no covariate, with yes indicating the presence of one or more precursor diseases.

\section{Exclusions and censoring}

Men registered with $\mathrm{MI}$, IS or HF before the date of study entry ( $n=1240,5 \%)$ and men who changed personal identification number during follow-up $(n<5,<1 \%)$ or had missing values on any of the covariates of interest $(N<300,1 \%)$ were excluded, leaving 25,436 men available for analysis (Fig. 1). We obtained vital status and emigration information from the Central Population Register. We were unable to retrieve information from hospital records for $\mathbf{2 8 0}$ men diagnosed with PCa and these men were not included in the study (15\%).

\section{Statistical methods}

First, hypothesised causal relations between confounders, mediators and CV event were identified in a diagram (Fig. 2). We then evaluated associations between these a priori determined confounders, mediators and the risk of first MI, IS or HF in the entire cohort (independent of $\mathrm{PCa}$ ) using univariate Cox proportional hazard models. We used age as underlying timescale and included death as competing risk along with sub-distribution hazards. The age-adjusted HRs for each potential confounder was reported along with the number of men, events, deaths and person-years for the 25,436 men in the cohort.

We followed all men from date of enrolment into the Diet, Cancer and Health study to the CV outcome of interest (MI, IS or $\mathrm{HF}$ ) or a censoring event (date of diagnosis of a competing CV event, emigration, non-CV death or end of study (31 December 2014)), whichever occurred first. First-line treatment was included as a time-varying exposure and was analysed using Cox proportional hazards models with stepwise adjustment for confounding variables, i.e., lifestyle, anthropometry and CV risk factors. Model 1 was adjusted for year of study entry. In Model 2, we added education, smoking, alcohol intake and physical activity. Model 3 further included anthropometry (BMI and waist circumference) and Model 4 included systolic and diastolic blood pressure, serum cholesterol and diabetes. In each analysis, the two other CV events were included as competing events together with death from other causes.

We estimated the cause-specific HR of each CV event stratified by first-line treatment among men with PCa compared with men without PCa adjusted for lifestyle, anthropometry and CV risk factors. We analysed temporal variation in HRs by splitting the time scale into three intervals according to time since diagnosis. As a sensitivity analysis, we adjusted for CV precursor diseases in Model 4 to see whetherpre-existing CV comorbidity confounded any association between PCa and CV events. We analysed the subdistribution hazards for each CV event without the two other events as competing events to see whether the direction of the association remained the same.

We tested the proportional hazards assumption in all analyses by inspecting $p$-values and plots of each covariate in all models based on scaled Schoenfeld residuals. We stratified all analyses by smoking status, due to continuous violation of the proportional hazards assumption. For the covariates BMI and MET, the proportionality assumption was violated in some of the models. These models were extended by including separate baseline hazards for each level of these confounders. However, the estimates for the exposures did not change after doing this and 


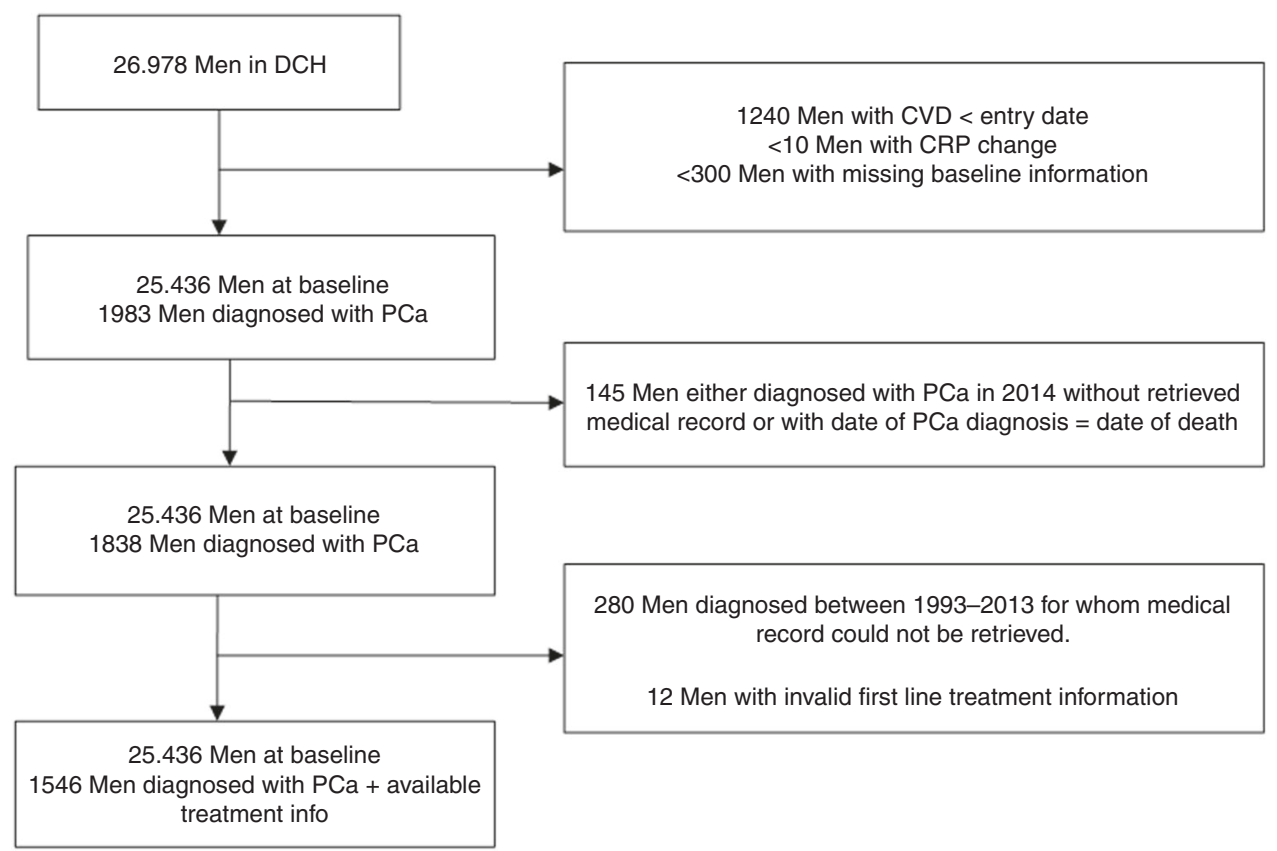

Fig. 1 Flow diagram showing the number of study participants in the Diet, Cancer and Health cohort, and the final study population in the study, 1993-2013

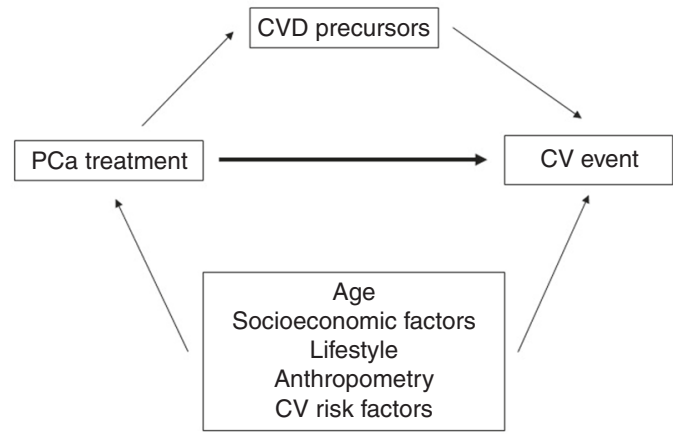

Fig. 2 Hypothesised causal relations between lifestyle, anthropometry, sociodemographics, cardiovascular risk factors and precursors, first-line PCa treatment and CV events

to be consistent throughout the analyses we used the original specification of the models with one baseline hazard. Cumulative incidences were calculated for each CV outcome and death from other causes (competing event) for each category of first-line treatment. The analyses were done in $\mathrm{R}$ version 3.3.3 with packages 'survival', 'ggplot2 and 'etm'. $27-29$

\section{RESULTS}

Of the 25,436 men participating in the Diet, Cancer and Health study, 1838 (7\%) were diagnosed with PCa during the median 18.5 years of follow-up accruing 387,032 person-years at risk. Only 342 (1\%) men were lost to follow-up due to migration. We observed $3 \%$ of the incident $1948 \mathrm{MI}, 4 \%$ of the 2144 IS and $4 \%$ of the 1547 HF among men diagnosed with PCa (Table 1). In the overall cohort, age-adjusted HRs for an incident CV event were increased for adverse exposure to lifestyle, anthropometry and CV risk factors (Table 1). The majority (>85\%) of men treated with curative intent were diagnosed with localised disease, which was only the case for about $20 \%$ of men receiving palliative care (Table 2). Further, more than half the men in palliative care had Gleason score $>8$ and $75 \%$ had a PSA level above 20 at time of diagnosis
(Table 2). Among the 1546 PCa patients with available treatment information, $9 \%$ were in active surveillance and $15 \%$ in watchful waiting, whereas $45 \%$ had received treatment with curative intention and $31 \%$ received palliative care as first-line treatment. We observed increased HRs for IS and HF among men in palliative treatment (adjusted HRs of $2.09,95 \% \mathrm{Cl} 1.49-2.93$ and HR 2.05, 95\% Cl 1.43-2.94, respectively) (Table 3). The adjusted causespecific HRs did not differ by time since diagnosis among men in first-line palliative treatment nor in any other treatment group. Including the CV precursors resulted in similar estimates (data not shown). In the sensitivity analysis, we analysed CV event rates without the other two outcomes as competing risks. Results showed the same direction of associations as the main analysis (data not shown). In the time split analysis, we observed that HRs for both IS and HF were increased already within 2 years of diagnosis and were so throughout follow-up. Estimates for IS increased throughout follow-up (HR 1.37, 95\% Cl 0.71-2.63 within 2 years post diagnosis and $\mathrm{HR} 3.03,95 \% \mathrm{Cl} 1.71-5.36$ beyond 5 years of diagnosis) (Table 3 ).

Cumulative incidence curves of $\mathrm{CV}$ events and non-CV death showed that the specific distribution by CV event varied (Fig. 3). The most pronounced difference was found for IS beyond 2.5 years post diagnosis in men receiving first-line palliative care.

\section{DISCUSSION}

We observed a twofold higher rate of both IS and HF among men treated with first-line palliative treatment for PCa compared with PCa-free men. The risk was increased since initiation of first-line treatment and for stroke we observed an increase in the HR rate over time. Adjusting for pre-cancer lifestyle factors, anthropometry and $\mathrm{CV}$ risk factors did not affect the increased risk. Among men treated curatively or conservatively for their $\mathrm{PCa}$, we found no statistically significant difference in risk for either MI, IS or HF compared with men without PCa.

The increased rate for $\mathrm{HF}$ in men receiving first-line palliative treatment for PCa compared with cancer-free men has also been observed in a population-based Swedish study. In this study, men with incident PCa undergoing endocrine treatment (i.e., antiandrogens (AAs), orchiectomy, gonadotropin-releasing hormone 
Table 1. Number of men in the study population, person-years at risk, number of events and age-adjusted HRs for first CV event

\begin{tabular}{|c|c|c|c|c|c|c|c|c|}
\hline \multirow[t]{2}{*}{ Baseline characteristics } & \multirow[t]{2}{*}{ No. of men } & \multirow[t]{2}{*}{ PYRS $^{\mathrm{a}}$} & \multicolumn{4}{|c|}{ No. of first major CV events } & \multirow{2}{*}{$\begin{array}{l}\text { No. of competing events } \\
\text { Deaths }\end{array}$} & \multirow{2}{*}{$\begin{array}{l}\text { Age-adjusted HR, HF, MI or stroke } \\
\text { HR }(95 \% \mathrm{Cl})\end{array}$} \\
\hline & & & All CV & MI & Stroke & $\mathrm{HF}$ & & \\
\hline Total & 25,436 & 397,085 & 5769 & 2005 & 2240 & 1632 & 3626 & - \\
\hline \multicolumn{9}{|l|}{ Educational attainment } \\
\hline Long (>12 years) & 6116 & 98,280 & 1136 & 386 & 463 & 310 & 722 & 1 \\
\hline Medium >7-12 years) & 10,627 & 167,328 & 2320 & 784 & 932 & 647 & 1431 & $1.21(1.12-1.29)$ \\
\hline Short ( $\leq 7$ years) & 8693 & 131,476 & 2313 & 835 & 846 & 675 & 1471 & $1.43(1.33-1.54)$ \\
\hline \multicolumn{9}{|l|}{ Lifestyle } \\
\hline \multicolumn{9}{|c|}{ Units of alcohol per week } \\
\hline 1-21 Drinks & 13,646 & 214,495 & 3115 & 1158 & 1174 & 844 & 1843 & 1 \\
\hline $22+$ Drinks & 8753 & 133,985 & 2067 & 633 & 842 & 630 & 1435 & $1.10(1.04-1.16)$ \\
\hline Abstainers & 3037 & 48,678 & 587 & 214 & 225 & 158 & 346 & $1.16(1.06-1.27)$ \\
\hline \multicolumn{9}{|l|}{ Smoking } \\
\hline Never & 6699 & 110,614 & 1217 & 400 & 506 & 331 & 594 & 1 \\
\hline Former & 8695 & 139,721 & 1803 & 623 & 681 & 533 & 990 & $1.11(1.04-1.20)$ \\
\hline Current & 10042 & 146750 & 2749 & 982 & 1054 & 768 & 2040 & $1.74(1.63-1.86)$ \\
\hline \multicolumn{9}{|l|}{ Physical activity } \\
\hline \multicolumn{9}{|l|}{ MET-score in quartiles ${ }^{c}$} \\
\hline$>43.5$ & 6273 & 99,786 & 1424 & 488 & 562 & 398 & 928 & 1 \\
\hline$>25.5-43.5$ & 6332 & 102,348 & 1355 & 468 & 535 & 376 & 888 & $0.94(0.87-1.01)$ \\
\hline $13.5-25.5$ & 6473 & 99,439 & 1397 & 522 & 508 & 406 & 864 & $0.99(0.92-1.06)$ \\
\hline$\leq 13.5$ & 6358 & 95,511 & 1593 & 527 & 636 & 452 & 944 & $1.19(1.10-1.27)$ \\
\hline \multicolumn{9}{|l|}{ Anthropometry } \\
\hline \multicolumn{9}{|l|}{ BMI } \\
\hline Normal weight $(<25)$ & 9017 & 143,399 & 1663 & 555 & 733 & 402 & 1371 & 1 \\
\hline Overweight (25-30) & 12,685 & 199,072 & 2964 & 1079 & 1134 & 806 & 1661 & $1.27(1.20-1.35)$ \\
\hline Obese $(>30)$ & 3734 & 54,613 & 1142 & 371 & 374 & 424 & 592 & $1.85(1.72-2.00)$ \\
\hline \multicolumn{9}{|c|}{ Waist circumference in quartiles } \\
\hline$<96$ & 7038 & 110,232 & 1478 & 489 & 634 & 378 & 1141 & 1 \\
\hline $96-100$ & 6637 & 105,003 & 1433 & 527 & 550 & 383 & 880 & $1.02(0.95-1.09)$ \\
\hline$>100-104<$ & 5799 & 91,115 & 1296 & 478 & 518 & 322 & 765 & $1.07(0.99-1.15)$ \\
\hline$>105$ & 5962 & 90,734 & 1562 & 511 & 539 & 549 & 838 & $1.32(1.23-1.41)$ \\
\hline \multicolumn{9}{|c|}{ Cardiovascular risk factors at baseline } \\
\hline \multicolumn{9}{|c|}{ Systolic blood pressure $(\mathrm{mmHg})$} \\
\hline$<120$ & 2684 & 44,540 & 387 & 143 & 157 & 94 & 336 & 1 \\
\hline $120-139$ & 9581 & 155,179 & 1763 & 661 & 648 & 488 & 1246 & $1.28(1.15-1.43)$ \\
\hline $140-160$ & 9041 & 139,665 & 2189 & 764 & 840 & 625 & 1325 & $1.69(1.51-1.88)$ \\
\hline$>160$ & 4130 & 57,700 & 1430 & 437 & 596 & 425 & 717 & $2.59(2.32-2.90)$ \\
\hline \multicolumn{9}{|c|}{ Diastolic blood pressure $(\mathrm{mmHg})$} \\
\hline$<80$ & 7665 & 124,473 & 1278 & 469 & 481 & 344 & 1059 & 1 \\
\hline $80-89$ & 9824 & 155,129 & 2169 & 794 & 799 & 620 & 1336 & $1.35(1.26-1.45)$ \\
\hline $90-100$ & 5682 & 85,663 & 1556 & 510 & 637 & 438 & 844 & $1.76(1.63-1.89)$ \\
\hline$>100$ & 2265 & 31,819 & 766 & 232 & 324 & 230 & 385 & $2.37(2.16-2.59)$ \\
\hline Serum cholesterol (mM & & & & & & & & \\
\hline$<5$ & 4315 & 67,868 & 863 & 227 & 359 & 295 & 688 & 1 \\
\hline $5-6.5$ & 14,053 & 221,436 & 3061 & 1072 & 1190 & 850 & 1935 & $1.07(0.99-1.15)$ \\
\hline$>6.5<7.5$ & 4965 & 76,332 & 1250 & 468 & 477 & 331 & 690 & $1.27(1.16-1.38)$ \\
\hline$>7.5$ & 2103 & 31,448 & 595 & 238 & 215 & 156 & 311 & $1.46(1.31-1.62)$ \\
\hline Diabetes & & & & & & & & \\
\hline No & 25,087 & 392,868 & 5599 & 1959 & 2178 & 1568 & 3558 & 1 \\
\hline Yes & 349 & 4216 & 170 & 46 & 63 & 64 & 66 & $2.89(2.47-3.38)$ \\
\hline Cardiovascular precurso & baseline $^{d}$ & & & & & & & \\
\hline 0 & 24,846 & 389,299 & 5488 & 1901 & 2163 & 1530 & 3538 & 1 \\
\hline $1+$ & 590 & 7785 & 281 & 104 & 78 & 102 & 86 & $2.44(2.16-2.75)$ \\
\hline
\end{tabular}

Number of men in the study population, person-years at risk, number of events and age-adjusted HRs for first CV event (MI, stroke, HF) with prostate cancer, education, lifestyle factors, anthropometric measures and cardiovascular risk factors and precursors in the 25,436 men in the Diet, Cancer and Health study. CV events cardiovascular events, HF heart failure, HR hazard ratio, MI acute myocardial infarction

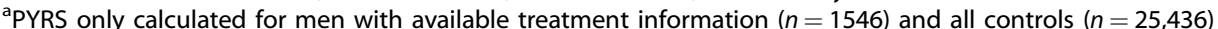

${ }^{\mathrm{b}}$ Total no of CV events does not equal the sum of MI, strokes and HF reported in the table, but is based on an algorithm where myocardial infarction overrules the other outcomes and stroke overrules heart failure

'The metabolic equivalent of task, i.e., a measure of energy cost of physical activities

${ }^{\mathrm{d}}$ Angina pectoris, transitional cerebral ischaemia or cardiomyopathy 


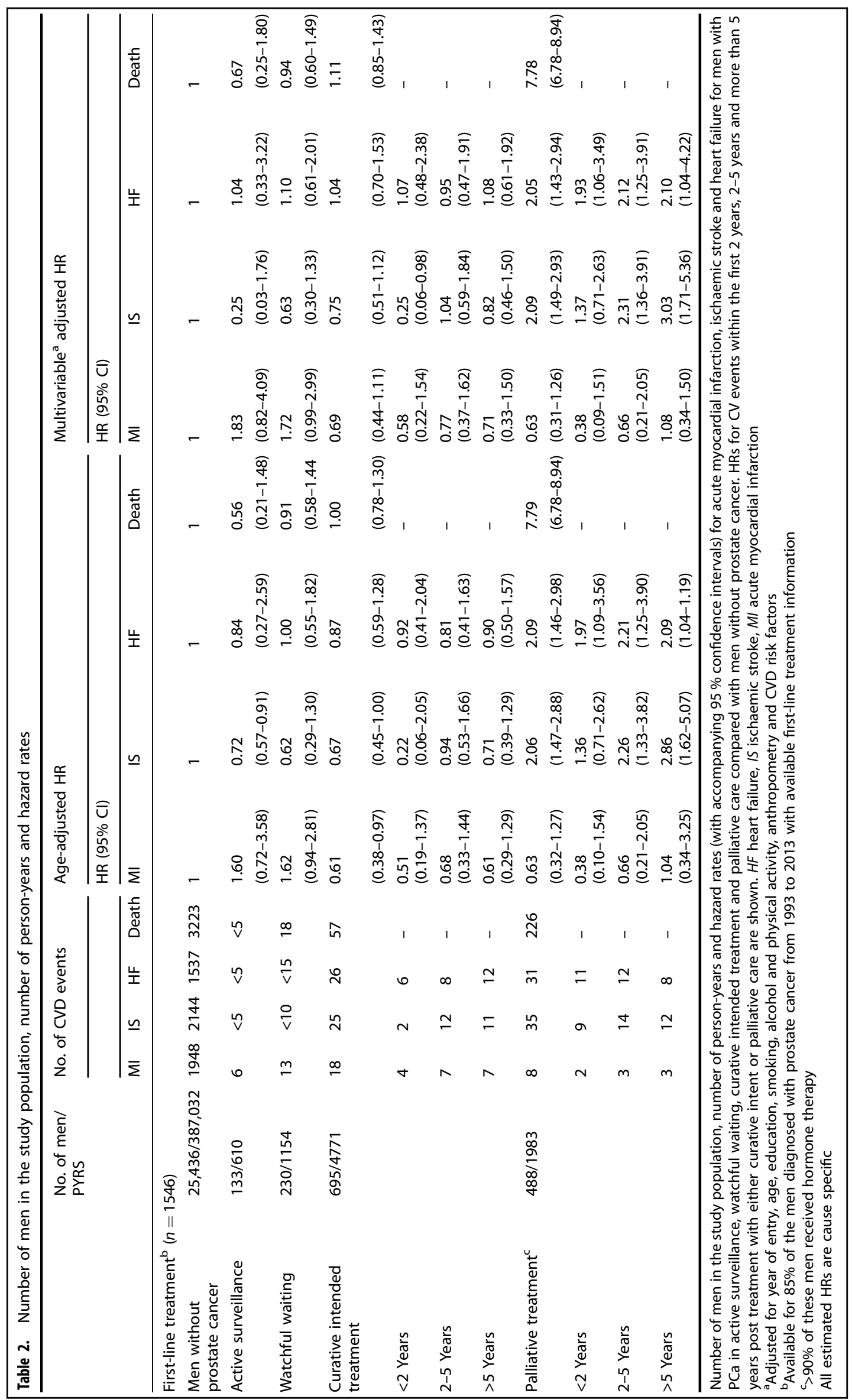


Table 3. Clinical characteristics for the 1546 men who were diagnosed with PCa from 1993 to 2013, who participated in the Diet, Cancer and Health study

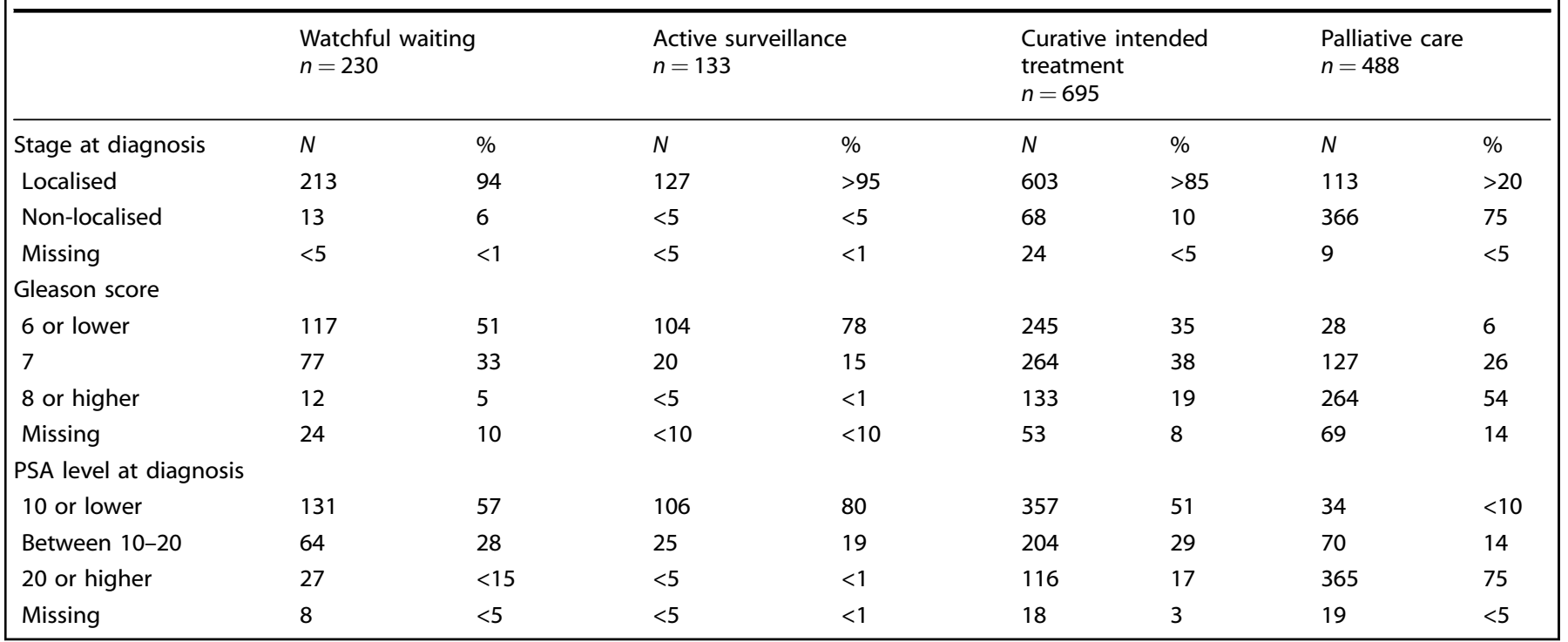

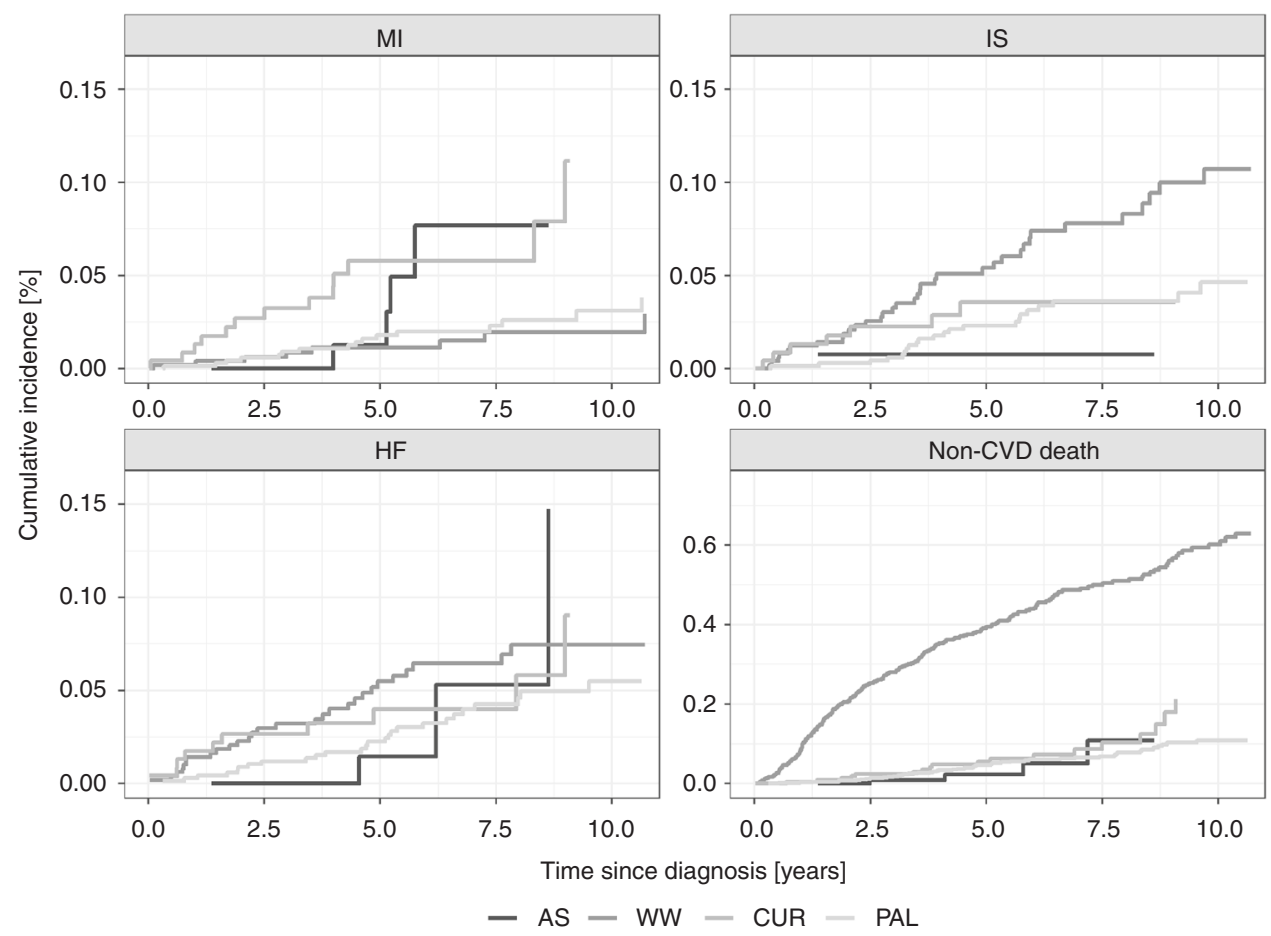

Fig. 3 Cumulative incidence plots for acute myocardial infarction, ischaemic stroke and heart failure, and death from other causes as competing event over time since diagnoses among 1546 men diagnosed with prostate cancer from 1993 through 2013

$(\mathrm{GnRH})$ agonists or combined $\mathrm{AA} / \mathrm{GnRH}$ agonist treatment) had an increased standardised incidence rate of 1.66 (95\% $\mathrm{Cl} 1.57-1.76)$ for HF compared with that expected based on the Swedish male background population without pre-existing circulatory disease. ${ }^{30}$ Unlike our study, they also found an increased risk of HF in the men who received curative treatment. Another Swedish matched cohort study compared the risk of HF between 41,362 ADT-treated PCa patients and age-matched controls. The HR was $1.22(95 \% \mathrm{Cl}$ 1.14-1.31) among men who received GnRH agonists and 1.12 (95\% Cl 1.04-1.21) among men who underwent surgical orchiectomy at time of diagnosis, but 0.83 (95\% Cl 0.71-0.97) among those receiving $A A$, when adjusting for history of CV disease, education, stage and statin use. This study used PCa-free men as reference group; however, this did not exclude men with pre-existing CV disease and they did not include lifestyle factors in the analyses. ${ }^{15}$ Regarding IS, we found a twofold increased rate among men managed on palliative care compared with PCa-free men, and to our knowledge the risk of IS in PCa patients has never been compared with the risk in men without PCa. One Danish cohort study including $>30,000$ men diagnosed with PCa observed an increased, although much lower adjusted HR for stroke of 1.19 (95\% Cl 1.06-1.35) among men managed on endocrine therapy (GnRH agonists, AA) but not in those undergoing orchiectomy compared with non-users of Androgen Deprivation Therapy 
(ADT). ${ }^{16}$ A US study of 22,310 men with non-metastatic PCa reported an increased risk of stroke or transient ischaemic attack (TIA) in men undergoing bilateral orchiectomy (Relative risk (RR) $1.77,95 \% \mathrm{Cl} 1.25-2.39)$ compared with non-ADT users, with patients age $>65$ years at diagnoses having an increased risk for stroke/TIA compared with the younger patients. ${ }^{31}$ We did not find an increased risk of $\mathrm{MI}$ in any treatment group when comparing with PCa-free men, which contrasts to large PCa cohort studies from United States and the United Kingdom including a minimum of 30,000 PCa patients and reporting risk estimates in the range of 1.09 to 1.40 (95\% Cl 1.01-1.49). ${ }^{30,32,33}$ To our knowledge, only one study has compared the risk of ischaemic heart disease, stroke and cardiomyopathy/HF between PCa patients and cancer-free men. ${ }^{14}$ The study found that among 10,932 PCa patients who survived diagnosis by 2 years, those with American Joint Committee on Cancer (AJCC) stage I/II and III had a lower CV risk (IRR 0.89, 95\% CI $0.84-0.95$ and IRR $0.79,95 \% \mathrm{Cl} 0.66-0.95$, respectively) compared with cancer-free men. The risk did not differ between PCa patients with stage IV and cancer-free men (IRR 1.03, 95\% Cl 0.78-1.35). ${ }^{14}$ However, the study lacked information on pre-diagnosis lifestyle, comorbidity and PCa treatment, and the study population was selected based on participants in an intensive screening programme through insurance coverage, which might have introduced selection bias as well as a high percentage of advanced tumours.

The strengths of our study include the use of high-quality registers. The Danish Cancer Registry holds accurate and almost complete recording of incident cancers ${ }^{23}$ and The NPR include information on both in- and outpatient contacts for CV events, which has been validated for research use. ${ }^{34,35}$ Further information on first-line treatment of PCa cases was obtained independently of the outcome of interest. We included all cancer stages in our analyses, thus providing a comprehensive perspective on how PCa treatment is associated with the risk of CV outcomes. Furthermore, our study had several methodological strengths. We made the comparison of men with PCa with a cohort of cancer-free men to disentangle the role of shared risk factors for PCa and CV disease. To the best of our knowledge, we are the first to include prospectively collected high-quality information on precancer lifestyle, objectively measured body composition, CV risk factors and precursor heart disease in the same study. We include both competing risk analysis and sub-distribution hazards to account for the significant competing events present in this study population. In our study, the results from sub-distribution hazard analyses aligned with the cause-specific hazards, which strengthens our interpretation that the estimated rates are likely to reflect a direct effect of PCa and treatment on the studied CV events. ${ }^{36}$ Our study has some limitations regarding information on outcomes of interest, treatment and selection into the study. Most cases of MI and IS will cause admission or other hospital contact. However, we acknowledge that our study results may still be prone to surveillance bias, i.e., that men diagnosed with PCa may have a higher probability of being diagnosed with particularly HF. When diagnosed with PCa, assessment of their stage of disease, symptom burden and severe comorbidity may result in confounding by indication. We excluded all men with pre-existing events, but still saw indications, albeit statistically nonsignificant of higher risk of $\mathrm{MI}$ among conservatively treated men and lower risk of $\mathrm{MI}$ among curatively treated men. No first-line treatments were associated with changes in risk over time after diagnosis. Among palliative treated men, the risk for both IS and HF was increased throughout the full follow-up period, despite a high non-CVD mortality, indicating that an effect of palliative first-line treatment on these CV outcomes may present from beginning of treatment and pointing to a need for early and targeted surveillance among these men. PCa patients initially managed on either active surveillance or watchful waiting may later have changed treatment strategy, and likewise men undergoing curatively intended therapy may subsequently have progressed during follow-up, potentially underestimating the difference between men who receive first-line palliative treatment and those who receive other first-line treatments. Watchful waiting is in general associated with older age, more comorbidity and shorter life expectancy than other treatments. This may explain the persistent findings among the conservatively treated men in watchful waiting of an increased, albeit statistically nonsignificant, risk of $\mathrm{Ml}$, whereas the risk for IS and HF tended to be very close to null or even reduced. As we excluded men with pre-existing CV events, this may have introduced more selection in terms of health in the group of patients allocated to watchful waiting than in the other treatment groups.

Some studies suggest that the risk of subsequent CV events correlates to the type of hormone therapy; ${ }^{16,37}$ however, we were not able to discriminate between subtypes of hormone therapy in the available data. This could efface within-group differences in the palliative group due to an underestimation for those receiving $\mathrm{GnRH}$ agonists and an overestimation among those who underwent bilateral orchiectomy. Contrary to expectations, the stepwise adjustment for lifestyle, anthropometry and CV risk factors did not affect the effect estimates markedly indicating that men with PCa in this cohort do not differ from the comparable men in terms of these $\mathrm{CV}$ risk factors. This may be because the men in the Diet, Cancer and Health cohort are somewhat healthier than the general population, thus resulting in less variation in risk factors, which is a well-known phenomenon in large-scale populationbased cohorts. ${ }^{38-40}$

We are aware that the results from this study population may not be generalisable to populations that have different baseline CV risks than Danish men and in settings with new chemo- and endocrine regiments available. Recent studies have demonstrated that combined chemo- and endocrine therapy, ${ }^{41}$ and addition of abiraterone to standard hormone therapy ${ }^{42}$ can improve survival in men with newly diagnosed metastatic PCa. The patients included in the present study were diagnosed through December 2013 and in the present period only few patients had chemotherapy in combination with first-line endocrine treatment. Abiraterone and second-generation AAs, i.e., enzalutamide and apalutamide, have not yet been approved in Denmark in combination with standard endocrine therapy in men with newly diagnosed metastatic disease. Thus, our data cannot provide information on the role of these therapies that would be both relevant and interesting to investigate in future studies, in PCa populations in relation to $\mathrm{CV}$ events. We encourage interpretation of the results to other settings with this in mind.

In summary, we found an increased risk of IS and HF among men who received first-line palliative treatment compared with men without cancer after adjusting for pre-cancer lifestyle, anthropometry and CV risk factors. As many of these men live with their PCa for a long time, it remains necessary to balance symptom relief and disease control with monitoring CV health in this patient group from the upstart treatment, in order to prevent or secure early management of CV events potentially affecting overall morbidity, healthrelated quality of life and ultimately mortality.

\section{ACKNOWLEDGEMENTS}

We thank all the participants from the Diet, Cancer and Health cohort, who made this study possible.

\section{AUTHOR CONTRIBUTIONS}

IRM, SBL, AKDH and SOD made the framework of hypothesised causal relations that guided the analyses. IRM and $\mathrm{AKDH}$ carried out the statistical analyses. KB provided clinical expertise. AT, SKK, CJ and SOD provided epidemiological expert knowledge. IRM took the lead in writing the manuscript. All authors provided critical feedback and helped shape the research, analysis and manuscript. 


\section{ADDITIONAL INFORMATION}

Supplementary information is available for this paper at https://doi.org/10.1038/ s41416-019-0468-8.

Competing interests: The authors declare no competing interests.

Funding: The project has been funded by Bjarne Saxhoff Foundation.

Ethics approval and consent to participate: The Diet, Cancer and Health study was approved by the regional ethical committees in Copenhagen and Aarhus ((KF) 11-037/01) and the present study by the Danish Data Protection Agency (2013-41-4232). All participants signed a written consent agreement at time of study enrolment.

Consent for publication: The manuscript does not hold any individual person data.

Data availability: The data used in the analyses for the manuscript is stored securely at the Danish Cancer Society Research Center and are available on request.

Note: This work is published under the standard license to publish agreement. After 12 months, the work will become freely available and the license terms will switch to a Creative Commons Attribution 4.0 International (CC BY 4.0).

Publisher's note: Springer Nature remains neutral with regard to jurisdictional claims in published maps and institutional affiliations.

\section{REFERENCES}

1. Engholm, G. F. J., Christensen, N., Kejs, A. M. T., Hertzum-Larsen, R., Johannesen, T. B., Khan, S. et al. NORDCAN: Cancer Incidence, Mortality, Prevalence and Survival in the Nordic Countries: Association of the Nordic Cancer Registries. Danish Cancer Society [Version 7.3 (08.07.2016)]. Available from: http://www.ancr.nu (2016).

2. Siegel, R. L., Miller, K. D. \& Jemal, A. Cancer statistics, 2017. CA Cancer J. Clin. 67, 7-30 (2017)

3. Center, M. M., Jemal, A., Lortet-Tieulent, J., Ward, E., Ferlay, J., Brawley, O. et al. International variation in prostate cancer incidence and mortality rates. Eur. Urol. 61, 1079-1092 (2012)

4. Outzen, M., Brasso, K., Martinussen, N., Christensen, J., Tjonneland, A., Friis, S. et al. Prostate cancer in Denmark 1978-2009--trends in incidence and mortality. Acta Oncol. 52, 831-836 (2013).

5. Shen, X. \& Kumar, P. Trade-off between treatment of early prostate cancer and incidence of advanced prostate cancer in the prostate screening era. J. Urol. 195, 1397-1402 (2016).

6. Conteduca, V., Di Lorenzo, G., Tartarone, A. \& Aieta, M. The cardiovascular risk of gonadotropin releasing hormone agonists in men with prostate cancer: an unresolved controversy. Crit. Rev. Oncol. Hematol. 86, 42-51 (2013).

7. de Haas, E. C., Oosting, S. F., Lefrandt, J. D., Wolffenbuttel, B. H. R., Sleijfer, D. T. \& Gietema, J. A. The metabolic syndrome in cancer survivors. Lancet Oncol. 11, 193-203 (2010).

8. Lenihan, D. J. \& Cardinale, D. M. Late cardiac effects of cancer treatment. J. Clin. Oncol. 30, 3657-3664 (2012).

9. Basaria, S., Muller, D. C., Carducci, M. A., Egan, J. \& Dobs, A. S. Hyperglycemia and insulin resistance in men with prostate carcinoma who receive androgendeprivation therapy. Cancer 106, 581-588 (2006).

10. Kintzel, P. E., Chase, S. L., Schultz, L. M. \& O'Rourke, T. J. Increased risk of metabolic syndrome, diabetes mellitus, and cardiovascular disease in men receiving androgen deprivation therapy for prostate cancer. Pharmacotherapy 28, 1511-1522 (2008).

11. Smith, M. R., Finkelstein, J. S., McGovern, F. J., Zietman, A. L., Fallon, M. A., Schoenfeld, D. A. et al. Changes in body composition during androgen deprivation therapy for prostate cancer. J. Clin. Endocrinol. Metab. 87, 599-603 (2002).

12. Smith, M. R., Lee, H. \& Nathan, D. M. Insulin sensitivity during combined androgen blockade for prostate cancer. J. Clin. Endocrinol. Metab. 91, 1305-1308 (2006).

13. Smith, J. C., Bennett, S., Evans, L. M., Kynaston, H. G., Parmar, M., Mason, M. D. et al. The effects of induced hypogonadism on arterial stiffness, body composition, and metabolic parameters in males with prostate cancer. J. Clin. Endocrinol. Metab. 86, 4261-4267 (2001).

14. Armenian, S. H., Xu, L., Ky, B., Sun, C., Farol, L. T., Pal, S. K., et al. Cardiovascular disease among survivors of adult-onset cancer: a community-based retrospective cohort study. J. Clin. Oncol. 34, 1122-1130 (2016).

15. O'Farrell, S., Garmo, H., Holmberg, L., Adolfsson, J., Stattin, P. \& Van Hemelrijck, M. Risk and timing of cardiovascular disease after androgen-deprivation therapy in men with prostate cancer. J. Clin. Oncol. 33, 1243-1251 (2015).
16. Jespersen, C. G., Norgaard, M. \& Borre, M. Androgen-deprivation therapy in treatment of prostate cancer and risk of myocardial infarction and stroke: a nationwide Danish population-based cohort study. Eur. Urol. 65, 704-709 (2014).

17. Leitzmann, M. F. \& Rohrmann, S. Risk factors for the onset of prostatic cancer: age, location, and behavioral correlates. Clin. Epidemiol. 4, 1-11 (2012).

18. Esposito, K., Chiodini, P., Capuano, A., Bellastella, G., Maiorino, M. I., Parretta, E. et al. Effect of metabolic syndrome and its components on prostate cancer risk: Meta-analysis. J. Endocrinol. Invest. 36, 132-139 (2013).

19. O'Donnell, C. J. \& Elosua, R. Cardiovascular risk factors. Insights from Framingham Heart Study. Rev. Esp. Cardiol. 61, 299-310 (2008).

20. de Koning, L., Merchant, A. T., Pogue, J. \& Anand, S. S. Waist circumference and waist-to-hip ratio as predictors of cardiovascular events: meta-regression analysis of prospective studies. Eur. Heart J. 28, 850-856 (2007).

21. Tjønneland, A., Olsen, A., Boll, K., Stripp, C., Christensen, J., Engholm, G. et al. Study design, exposure variables, and socioeconomic determinants of participation in Diet, Cancer and Health: a population-based prospective cohort study of 57,053 men and women in Denmark. Scand. J. Public Health 35, 432-441 (2007).

22. Pedersen, C. B. The Danish Civil Registration System. Scand. J. Public Health 39 (7_suppl), 22-25 (2011).

23. Gjerstorff, M. L. The Danish Cancer Registry. Scand. J. Public Health 39(7 Suppl), 42-45 (2011).

24. Karlsen, R. V., Larsen, S. B., Christensen, J., Brasso, K., Friis, S., Tjønneland, A. et al. PSA testing without clinical indication for prostate cancer in relation to sociodemographic and clinical characteristics in the Danish Diet, Cancer and Health Study. Acta Oncol. 52, 1609-1614 (2013).

25. Lynge, E., Sandegaard, J. L. \& Rebolj, M. The Danish National Patient Register. Scand. J. Public Health 39(7 Suppl), 30-33 (2011).

26. Helweg-Larsen, K. The Danish Register of Causes of Death. Scand. J. Public Health 39(7 suppl), 26-29 (2011).

27. Therneau, T. A Package for Survival Analysis in S [2.38]. Available from: https:// CRAN.R-project.org/package=survival (2015).

28. Wickham, H. ggplot2: Elegant Graphics for Data Analysis (Springer-Verlag, New York, 2009).

29. Team R. C. R: A language and environment for statistical computing. 0.99.896 ( $R$ Foundation for Statistical Computing, Vienna, Austria, 2017).

30. Van Hemelrijck, M., Garmo, H., Holmberg, L., Ingelsson, E., Bratt, O., Bill-Axelson, A. et al. Absolute and relative risk of cardiovascular disease in men with prostate cancer: results from the Population-Based PCBaSe Sweden. J. Clin. Oncol. 28, 3448-3456 (2010).

31. Azoulay, L., Yin, H., Benayoun, S., Renoux, C., Boivin, J. F. \& Suissa, S. Androgendeprivation therapy and the risk of stroke in patients with prostate cancer. Eur. Urol. 60, 1244-1250 (2011).

32. Gandaglia, G., Sun, M., Popa, I., Schiffmann, J., Abdollah, F., Trinh, Q.-D. et al. The impact of androgen-deprivation therapy (ADT) on the risk of cardiovascular (CV) events in patients with non-metastatic prostate cancer: a population-based study. BJU Int. 114(6b), E82-E89 (2014).

33. Keating, N. L., O'Malley, A. J. \& Smith, M. R. Diabetes and cardiovascular disease during androgen deprivation therapy for prostate cancer. J. Clin. Oncol. 24, 4448-4456 (2006)

34. Krarup, L. H., Boysen, G., Janjua, H., Prescott, E. \& Truelsen, T. Validity of stroke diagnoses in a National Register of Patients. Neuroepidemiology 28, 150-154 (2007).

35. Sundbøll, J., Adelborg, K., Munch, T., Frøslev, T., Sørensen, H. T., Bøtker, H. E., et al. Positive predictive value of cardiovascular diagnoses in the Danish National Patient Registry: a validation study. BMJ Open. 6, e012832, 2016.

36. Latouche, A., Allignol, A., Beyersmann, J., Labopin, M. \& Fine, J. P. A competing risks analysis should report results on all cause-specific hazards and cumulative incidence functions. J. Clin. Epidemiol. 66, 648-653 (2013).

37. Smith, M. R., Klotz, L., van der Meulen, E., Colli, E. \& Tanko, L. B. Gonadotropinreleasing hormone blockers and cardiovascular disease risk: analysis of prospective clinical trials of degarelix. J. Urol. 186, 1835-1842 (2011).

38. Larsen, S. B., Dalton, S. O., Schüz, J., Christensen, J., Overvad, K., Tjønneland, A. et al. Mortality among participants and non-participants in a prospective cohort study. Eur. J. Epidemiol. 27, 837-845 (2012).

39. Jacobsen, T. N., Nohr, E. A. \& Frydenberg, M. Selection by socioeconomic factors into the Danish National Birth Cohort. Eur. J. Epidemiol. 25, 349-355 (2010).

40. Nohr, E. A., Frydenberg, M., Henriksen, T. B. \& Olsen, J. Does low participation in cohort studies induce bias? Epidemiology 17, 413-418 (2006).

41. Sweeney, C. J., Chen, Y. H., Carducci, M., Liu, G., Jarrard, D. F., Eisenberger, M. et al. Chemohormonal therapy in metastatic hormone-sensitive prostate cancer. $N$. Engl. J. Med. 373, 737-746 (2015).

42. Fizazi, K., Tran, N., Fein, L., Matsubara, N., Rodriguez-Antolin, A., Alekseev, B. Y. et al. Abiraterone plus prednisone in metastatic, castration-sensitive prostate cancer. N. Engl. J. Med. 377, 352-360 (2017). 